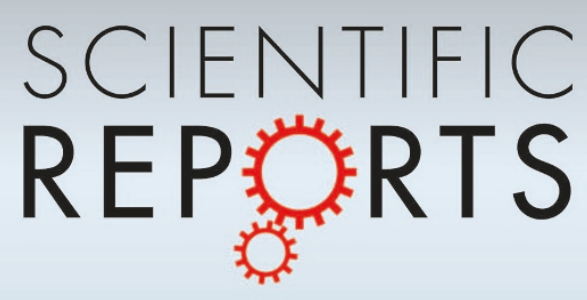

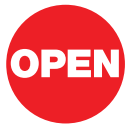

SUBJECT AREAS:

NANOPARTICLES

MECHANICAL AND STRUCTURAL PROPERTIES AND DEVICES

STRUCTURE OF SOLIDS AND

LIQUIDS

SURFACES, INTERFACES AND THIN FILMS

Received

15 October 2012

Accepted

30 November 2012

Published

18 December 2012

Correspondence and requests for materials should be addressed to X.X.Z. (xixiang. zhang@kaust.edu.sa)

\section{Unraveling the Atomic Structure of Ultrafine Iron Clusters}

\author{
Hongtao Wang ${ }^{1,2}$, Kun Li ${ }^{2}$, Yingbang Yao ${ }^{2}$, Qingxiao Wang ${ }^{2}$, Yingchun Cheng ${ }^{3}$, Udo Schwingenschlögl ${ }^{3}$, \\ Xi Xiang Zhang ${ }^{2} \&$ Wei Yang'
}

${ }^{1}$ Institute of Applied Mechanics, Zhejiang University, Hangzhou 310027, China, ${ }^{2}$ Advanced Nanofabrication, Imaging and Characterization Core Lab, King Abdullah University of Science and Technology, Thuwal 239955, Kingdom of Saudi Arabia, ${ }^{3}$ Materials Science and Engineering Department, King Abdullah University of Science and Technology, Thuwal 239955, Kingdom of Saudi Arabia.

Unraveling the atomic structures of ultrafine iron clusters is critical to understanding their size-dependent catalytic effects and electronic properties. Here, we describe the stable close-packed structure of ultrafine Fe clusters for the first time, thanks to the superior properties of graphene, including the monolayer thickness, chemical inertness, mechanical strength, electrical and thermal conductivity. These clusters prefer to take regular planar shapes with morphology changes by local atomic shuffling, as suggested by the early hypothesis of solid-solid transformation. Our observations differ from observations from earlier experimental study and theoretical model, such as icosahedron, decahedron or cuboctahedron. No interaction was observed between $\mathrm{Fe}$ atoms or clusters and pristine graphene. However, preferential carving, as observed by other research groups, can be realized only when Fe clusters are embedded in graphene. The techniques introduced here will be of use in investigations of other clusters or even single atoms or molecules.

U ltrafine clusters, containing less than a few hundred atoms (diameters of 1-3 nm), have size-dependent effects in catalytic processes and electronic structures ${ }^{1,2}$. The starting point to explore these properties is to unravel the atomic structures of the ultrafine clusters. Clusters with diameters larger than $3 \mathrm{~nm}$ have been extensively studied by atomic resolution transmission electron microscopy (TEM) over the past few decades ${ }^{3-13}$. However, unambiguous determination of the three-dimensional (3D) atomic structure of ultrafine clusters remains a challenge. Because of the active nature of low-coordinated particles ${ }^{14,15}$, the free energy may be overwhelmed by the chemical bonding to the support during TEM imaging, which leads to substantial changes in the atomic arrangement $t^{2,5,8,9,12,16}$. Besides, the elastic strain induced through the contact also causes structural modifications to the small energy barriers in various configuration $s^{5-10}$. The consequences of these factors are further exaggerated by the excitation from the high-energy (beyond $200 \mathrm{keV}$ ) incident electron beam. Heat absorption from inelastic electron-nucleus scattering leads to structural instability and fluctuations via atomic rearrangement. The consequential morphology change has long been the subject of debate. Some argue that it occurs through a melting-recrystallization process, whereas others suggest that it is through solid-solid transformation $^{1,3,7-11}$. Although high resolution TEM imaging benefits from the high-energy electron source, and the uniform and low background of amorphous substrates, the high transferred energy and the unknown surface structure of the support significantly affect the behavior of ultrafine clusters and introduce extra complexity in resolving atomic structures under electron irradiation. Until now, there is no clear-cut TEM observation showing direct evidence of the atomic structure of ultrafine clusters.

In this paper, we seek to resolve with atomic resolution the three-dimensional shape, atomic arrangement and structural transformation of ultrafine Fe clusters on graphene supports by using monochromated and aberration-corrected low-voltage TEM. The ultrafine Fe clusters, containing a few tens to hundreds of atoms, have close-packed atomic structures and take stable planar shapes of equilateral triangles, isosceles trapezoids or parallelograms with internal angles of $60^{\circ}$ or $120^{\circ}$. The transition between configurations is assisted by local atomic shuffling, which is different from any known mechanism. Melting-like behavior was also observed due to the large disorder induced by bonding to the random dangling $\mathrm{C}$ atoms along the graphene edge, which indicates that contradictions in early TEM studies may arise from unknown interactions in the substrates ${ }^{2,5,7,10,11}$. We believe that detailed structural information will be useful to investigating the catalytic reactions of Fe clusters, which are now finding more and more applications because of their low cost and environmental benignity. We 
also note that the techniques introduced here will be of use in investigations of other clusters or even single atoms or molecules ${ }^{16-19}$.

\section{Results}

Free-standing graphene was used as the substrate (Figure S1a), which is known to be a strong and thin continuous film with a honeycomb lattice. The excellent electrical and thermal conductivity of grapheme minimize both the charging and heating effects. In addition, its chemical inertness induces the least disturbance to the cluster via van der Waals interactions. We deposited the iron clusters by pulsed laser deposition in an ultrahigh vacuum chamber. Particles, ranging from single atoms to clusters with a few hundred atoms, were ejected from the target surface during laser ablation with a strong forwarddirected velocity. The bombardment induced various defects to the free-standing graphene ${ }^{18}$, which helped to bind the clusters and inhibit agglomeration. The as-prepared sample was transferred to a TEM equipped with monochromator and objective lens spherical aberration corrector, which enabled a resolution better than $1.4 \AA$ at $60 \mathrm{kV}$ (Figure S1b,c). The maximum energy transferred to $\mathrm{C}$ atoms was $11.6 \mathrm{eV}$, far below the knock-on threshold energy $(17 \mathrm{eV})$ for ejection of an in-lattice atom. Electron energy loss spectra on the clusters were collected to identify the chemical composition (Fig. S2). The indiscernible $\mathrm{O}$ edge, as compared to the Fe edge, indicates no oxidation formation. The experimental details are further described in the method section.

Figure $1 \mathrm{a}-\mathrm{f}$ show a series of TEM micrographs extracted from Movie S1, capturing the structural evolution of a typical Fe cluster sitting on a monolayer graphene encircled by bilayer regions of gra-

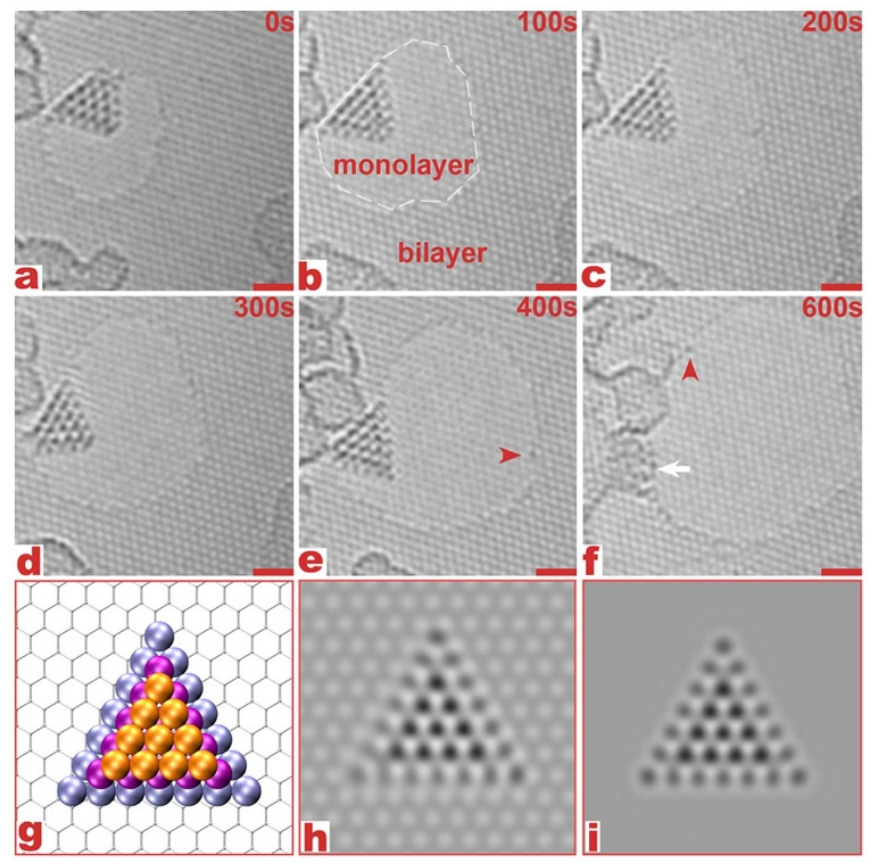

Figure 1 The structural evolution of an ultrafine Fe cluster under electron irradiation. (a-f) An aberration-corrected TEM image sequence of a cluster sitting on a graphene support, which is divided into two regions of monolayer and bilayer graphene. The white dashed line delineates the boundary in (b). Scale bar: $1 \mathrm{~nm}$. See also the recorded video in supplementary Movie S1. The red arrow heads in (e) and (f) point to single Fe atoms trapped on the edge. The cluster in (f), as marked by the white arrow, combines with the adsorbate on graphene and no longer retains its shape of a regular triangle. (g) An atomic model of the cluster with 53 atoms in a three-layer hexagonal close-packed structure.

Corresponding TEM simulations of the model (h) with and (i) without a monolayer graphene support were performed by using the software MacTempasX incorporated with the multislice method. phene over a time span of $10 \mathrm{~min}$. The cluster takes the shape of a regular triangle with one side in contact with the zigzag edge of the top graphene layer. The covalent bonding between the metal atoms and the graphene edge, as determined by density functional theory (DFT), prevents translational motion of the cluster and, therefore, improves TEM imaging through the use of a slow-scan, high-resolution CCD camera. As shown in the figure, the iron atoms assemble into a close-packed structure, similar to the piling of cannonballs with the highest packing density, which is different from the bodycentered cubic (BCC) lattice in its bulk form. Two different stacking sequences of the close-packed atomic layers give the same packing density, but result in different symmetries, i.e., face-centered cubic (FCC) and hexagonal close-packed (HCP) stacking. Relative to a reference layer with positioning $A$, two more positionings, $B$ and $C$, are possible (Fig. S3). FCC stacking is in the order of $A B C A B C$, while HCP stacking has the sequence $A B A B$. To distinguish between the two stacking orders, high-resolution TEM image simulations were performed for various packing structures with 1 to 4 atomic layers (Fig. 1 g-i, Fig. S4 d-f, Fig. S5). Only the simulation of the three-layer HCP structure (Fig. 1g-i) matches the TEM observations. Close comparison between Fig. $1 \mathrm{~h}$ and 1i suggests that the background, induced by the monolayer graphene, has no discernible interference with the Fe clusters. Direct counting of the TEM image indicates that the intact edge consists of $7 \mathrm{Fe}$ atoms, which gives 53 atoms in total for the cluster. The nearest neighbor distance is measured to be $2.7 \pm 0.15 \AA$ by using the inherently built atomic scale of the $\mathrm{C}-\mathrm{C}$ bond length of $1.42 \AA$ (Inset to Fig. S1c). This value is close to the nearest neighbor distance $(2.58 \AA$ ) of bulk FCC iron (PDF 52 0513).

Other configurations, such as a bilayer triangle and a trilayer trapezoid with HCP or FCC stacking, have been identified in our TEM study using graphene as a support (Fig. 2a-i, Fig. S6, Fig. S7). Surprisingly, all ultrafine Fe clusters have planar close-packed structures, which is different from the predicted equiaxial shape, such as an icosahedron, decahedron or cuboctahedron, by both DFT and molecular dynamics studies ${ }^{20}$. The experimentally observed unique shape may be attributed to the HCP structure in which the basal plane has the lowest surface energy. Iron clusters are attached to either adsorbates (Fig. 2a, Fig. S6c-f, Fig. S7) or graphene edges (Fig. 2b-c, Fig. S6a,b). Boundary Fe atoms are easily rearranged to accommodate the shape of the edge, which may be due to the relatively rigid $\mathrm{C}-\mathrm{C}$ bonds and the high binding energies between the metal atoms and graphene edges ${ }^{19}$. In contrast, the free edges of the clusters are generally straight and along the $\langle 110\rangle$ close-packed directions (Fig. 2j).

\section{Discussion}

A striking feature is the stability of the ultrafine iron clusters under electron irradiation with a beam current density of about $7 \times 10^{6}$ $\mathrm{e} \cdot \mathrm{s}^{-1} \cdot \mathrm{nm}^{-2}$ (i.e. $100 \mathrm{~A} / \mathrm{cm}^{2}$ ). In a previously reported TEM investigation of metal clusters, a structural fluctuation on the time scale of seconds was generally observed among different configurations, such as icosahedra, decahedra or multi-twinned structures ${ }^{2,3,9}$. Numerous hypotheses were proposed to explain the non-static nature, including soft configurational energy surfaces and quasi-molten states ${ }^{5,8,11,21}$. It has even been suggested that the structural fluctuation is intrinsic due to the finite size effect ${ }^{5,22}$. In our sample, the cluster almost retains the shape of a triangle for a time span of $\sim 8 \mathrm{~min}$ (Movie S1), except for local rearrangements of atoms near the vertex when it comes into contact with the irregular graphene edge (Fig. 1c-e). It is known that the irradiation effect comes into play only if the maximum transferred energy via electron-nucleus scattering is higher than the threshold energy in removing an atom from its original site ${ }^{23}$. Under the current imaging conditions, the maximum transferred energy is $2.5 \mathrm{eV}$ for $\mathrm{Fe}$ atoms. The threshold in the displacement of atoms is related to the cohesive energy of small iron clusters, which 


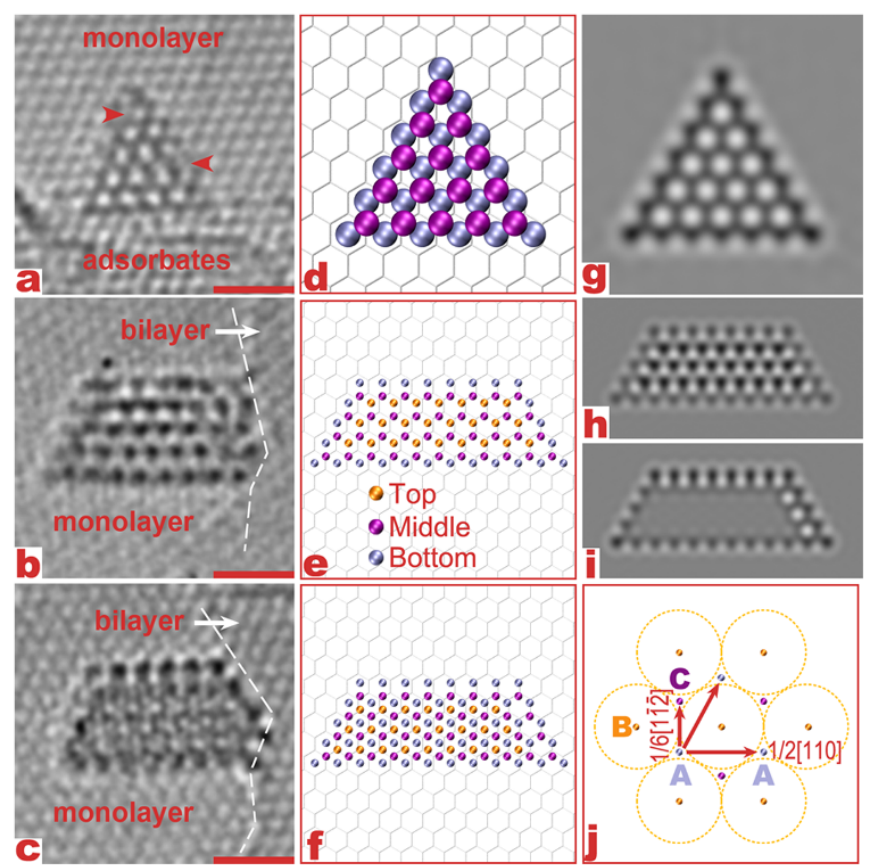

Figure $2 \mid$ Morphologies and structures of ultrafine Fe clusters.

(a) Bilayer triangular, (b) trilayer trapezoid $\mathrm{HCP}$ and (c) trilayer trapezoid FCC Fe clusters supported by graphene. (b) and (c) show the same Fe cluster but different stacking orders. Vacancies in the cluster in (a) are indicated by red arrow heads. The white dashed lines in (b) and (c) delineate the boundaries between monolayers and bilayers of graphene. Scale bar: $1 \mathrm{~nm}$. (d-f) Corresponding atomic models showing the stacking configurations. There are 36 atoms in (d) and 112 atoms in (b) and (c). (g-i) The corresponding TEM simulations of models in (d-f). (j) A schematic diagram showing the slip systems in HCP or FCC stacking. $<110>$ is the in-plane close-packed direction.

has been found to monotonically increase with cluster size and is about $3 \mathrm{eV}$ for an $\mathrm{Fe}$ cluster with 55 atoms $^{20}$. It is therefore not surprising that electron irradiation does not lead to substantial morphological changes in ultrafine iron clusters, given that no other factors, such as charging or beam heating, come into play. Also, there is no discernible interaction with the pristine graphene. Iron clusters have been observed to move freely on perfect graphene once it is detached from the edge due to the transferred momentum from high-energy electrons. No defects were introduced to the originally perfect graphene under the iron cluster after prolonged irradiation (Fig. 1f). In contrast, strong bonding has been observed between $\mathrm{Fe}$ atoms and dangling $\mathrm{C}$ atoms, which confines the movements of $\mathrm{Fe}$ atoms to the edge (Fig. 1e, f).

To be reconciled with earlier TEM observations ${ }^{5,9}$, the interactions between particles and the support should be taken into account. Though these interactions have long been known to be important in determining both morphologies and structures ${ }^{2,5}$, they remain nearly untouched in most experiments, theoretical modeling and simulations, due to the involved complexities and uncertainties in the surface structures of common TEM grid membranes made of amorphous carbon, $\mathrm{SiO}_{2}, \mathrm{SiN}_{x}$ and $\mathrm{Al}_{2} \mathrm{O}_{3}$. Light elements and amorphous structures are useful in providing low and uniform backgrounds. However, the thickness of the membrane, ranging from 5 to $50 \mathrm{~nm}$, conceals the detailed atomic bonding information between ultrafine clusters and substrates. Also, the light elements, especially in the amorphous state, are liable to suffer from bond breaking, atomic displacement or sputtering effects from electron irradiation due to the much higher transferred energy ${ }^{24}$, which renders the nature of surface structures dynamic and cannot be captured in common TEM observations. The instabilities can be transferred to the metal atoms or clusters by forming or breaking bonds at the surface, as demonstrated in our investigation of the dynamic behaviors of single $\mathrm{Au}$ atoms on a graphene edge ${ }^{17}$. The unstable structure of ultrafine Fe clusters on defective graphene clearly reveals the strong influence imposed by substrates (Movie S2). Figure 3 shows a series of extracted video frames. Structural distortion starts from the vertex of the iron triangle (Fig. 3b-c, indicated by an arrow), implying preferential bonding to the defect underneath. For about $1 \mathrm{~min}$, the iron cluster totally loses its original shape and embeds itself into a hole that was catalytically etched under electron irradiation (Fig. 3d-f). The dangling $\mathrm{C}$ atoms on the edge have lower binding energies, ranging from $5.5 \mathrm{eV}$ to $10.5 \mathrm{eV}^{17}$, than the maximum transferred energy of $11.6 \mathrm{eV}$, and, therefore, have a non-zero displacement rate. The hole is enlarged by prolonged irradiation (Fig. $3 g-i$ ), leading to dynamic contact with the iron cluster. The preferential bonding to the dangling $\mathrm{C}$ atoms changes both the morphology and atomic arrangement of the cluster on a time scale of a few seconds (Movie S2). The snapshots in Fig. 3 capture both ordered and disordered configurations, termed "molten" and "crystallized" states in the literature ${ }^{2,5,9-11}$. We note that more stable structures with the close-packed configuration are easily formed on the zigzag edge (Fig. 3i), which is known to be relatively stable under electron irradiation $^{25,26}$.

It is interesting to observe that the iron cluster preferentially carves the graphene along the armchair direction $(<110>)$ if it is embedded in the graphene (Fig. 4). We note that both the hydrogenationassisted Fe channeling and the thermally activated Ag etching are along the zigzag directions $s^{27-29}$, suggesting that different operating mechanisms are at work. In the high vacuum ambient environment of a TEM column, no $\mathrm{H}$ or $\mathrm{O}$ is expected to assist the preferential etching process, as reported in Ref 30 and 31. The underlying mechanism may reside in the strong $\mathrm{C}$-Fe bonding and associated catalytic effect, which lowers the vacancy formation energy as according to results from $\mathrm{DFT}^{32}$. As revealed by Movie S3, the structure evolves

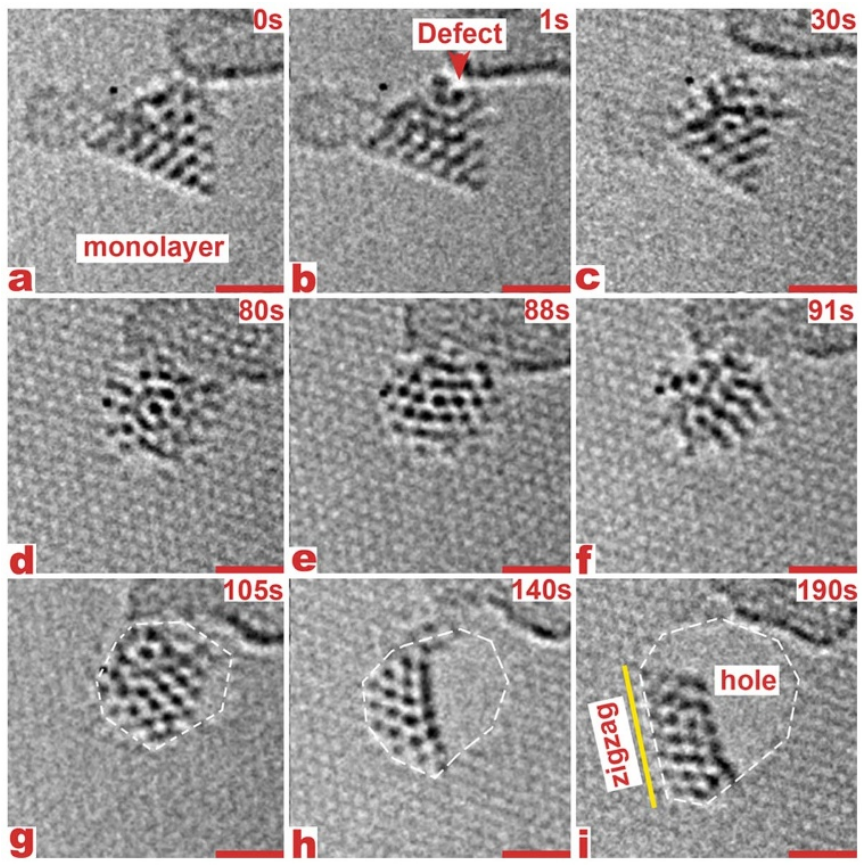

Figure 3 Interactions between ultrafine Fe clusters and defective graphene. (a-i) An aberration-corrected TEM image sequence of the structural evolution of an ultrafine Fe cluster sitting on a defective monolayer graphene. The red arrow head in (b) indicates that a defect may exist underneath the cluster. The white dashed lines in (g-i) delineate the boundaries of a hole. The yellow line indicates in (i) the zigzag direction. Scale bar: $1 \mathrm{~nm}$. See also the recorded video in supplementary Movie S2. 

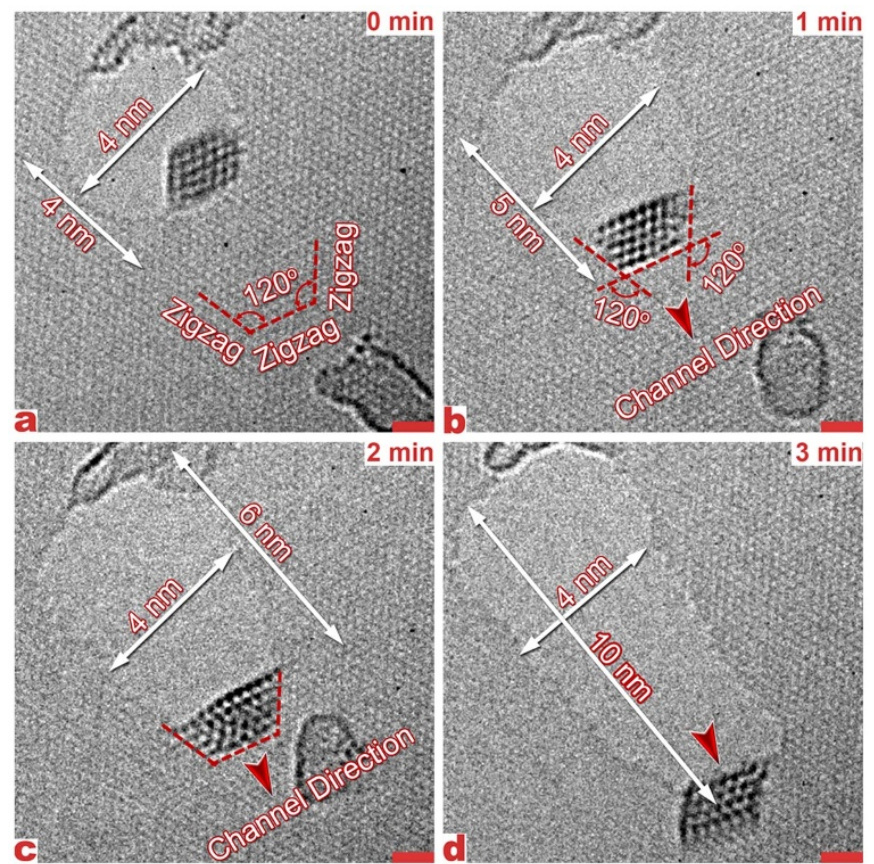

Figure $4 \mid$ Channeling of Fe clusters embedded in graphene. (a-d) An aberration-corrected TEM image sequence of the structural evolution of an ultrafine Fe cluster embedded in a monolayer of graphene. The red arrow heads in (b-c) indicate the channel direction. Scale bar: $1 \mathrm{~nm}$. See also the recorded video in supplementary Movie S3.

under electron irradiation, leading to bonding formation or breakage. Carbon atoms are prone to be removed from the front interface and replaced by Fe atoms. Row-by-row removal of the front atoms leads to preferential etching along the $\langle 110\rangle$ direction. The channel edge is not atomically smooth, though it is along certain low-energy crystallographic orientations. The roughness comes from the vibration due to the irradiation-induced wiggling motion of the Fe clusters.

A deformed lattice is also observed due to straining (Fig. S6b) or the curved boundary (Fig. S6c), which is tolerated because of the negligible contribution of elastic energy to the total energy on such a small scale. The stability of the cluster is mostly determined by the graphene edges or adsorbates. The major effect from electron irradiation at low voltage is to overcome energy barriers and enhance atomic diffusion to achieve more stable configurations. The edge or vertex Fe atoms have lower coordination numbers and are relatively easily displaced from the original sites to nucleate surface vacancies in the clusters, as indicated by the arrows in Fig. 2a and Fig. S6e-f. Also, the stacking order may be altered by the continuous agitation from the incident beam. Figures $3 \mathrm{~b}$ and $\mathrm{c}$ show snapshots of the same Fe cluster but with HCP and FCC stacking, respectively. Due to the limited temporal resolution, no information can be obtained from uncovering the detailed transition process. However, it is well known that a stacking fault can be easily generated by the slipping of adjacent layers by a relative vector of $1 / 6<112>$ (Fig. $2 j$ ), which may imply a collective motion of atoms on the small scale.

Based on our TEM observations of graphene-supported ultrafine Fe clusters, the well-defined geometries may lead to the conclusion that energy minimization dominates the shape of the structures. Using macroscopic concepts as a guide, the total energy can be written as

$$
U_{\text {tot }}=u_{\mathrm{B}} N_{\mathrm{B}}+u_{\mathrm{S}} N_{\mathrm{S}}+u_{\mathrm{E}} N_{\mathrm{E}}+u_{\mathrm{V}} N_{\mathrm{V}}+u_{\mathrm{I}} N_{\mathrm{I}}
$$

where $u_{\alpha}$ is the specific energy per atom with subscript " $\alpha$ " being B (bulk), S (surface), E (edge), V (vertex) and I (interface), and $N_{\alpha}$ is the corresponding number of atoms. The adhesion energy, as indicated by the interfacial term $\left(u_{\mathrm{I}} N_{\mathrm{I}}\right)$, characterizes the substrate effect in inducing metastable phases in metal clusters. It is noted that the graphene surface put a less constraint than other typical well-defined metal oxide surfaces, such as $\mathrm{MgO}(001)^{33-35}$. Early TEM observations ${ }^{34}$ have revealed local epitaxial relation between ultrafine metal clusters and the oxide substrate surface. Consequently different atomic arrangements may present with or without a substrate, as suggested by DFT calculations ${ }^{33,36}$. Our DFT study shows that the close-packed structure of ultrafine Fe clusters is metastable with or without the presence of graphene substrates (Fig. S8). This is consistent with our TEM observation that the ultrafine Fe cluster takes the same structure when sitting on the edge of the hole in a graphene (Fig. S6a). For regular planar shapes, the equal-energy configurations
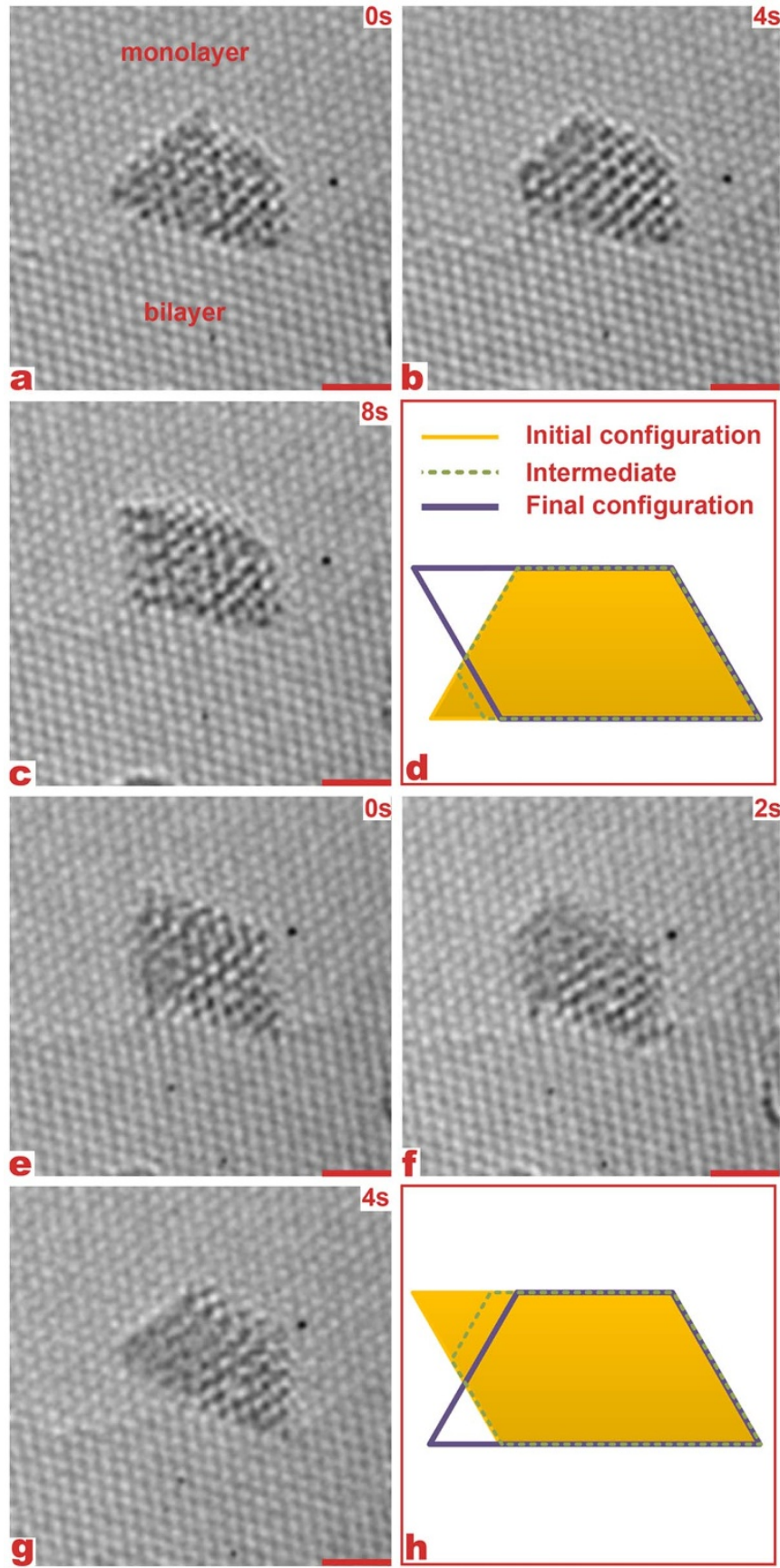

Figure $5 \mid$ Morphology changes in ultrafine Fe clusters. (a-c) A transition from trapezoid to parallelogram and (e-g) vice versa. Scale bar: $1 \mathrm{~nm}$. See also the recorded video in supplementary Movie S4. (d) and (h) illustrate the corresponding processes. 
can be identified, as shown in Figs. $2 \mathrm{a}$ and $\mathrm{c}$, or $2 \mathrm{e}$ and g. The transition from an isosceles trapezoid to a parallelogram and vice versa are clearly caused by local atomic shuffling on a time scale of seconds (Fig. 5, see also Movie S4), while the other part remains undisturbed during the process. This observation is consistent with the solid-solid transformation hypothesis ${ }^{10}$.

In summary, we unambiguously unraveled the atomic structure of ultrafine Fe clusters on graphene supports for the first time. Its monolayer thickness, chemical inertness, mechanical strength, and electrical and thermal conductivity render graphene an ideal substrate for high-resolution TEM imaging. Moreover, the state-ofthe-art aberration-corrected TEM technology enables electron microscopy with atomic resolution at low voltage, which further minimizes the disturbance and helps to uncover the atomic structures in the original state. Taking advantage of both the grapheme support and the technology made it possible that the ultrafine Fe clusters, containing only a few tens to hundreds of atoms, were rather stable under high-flux electron irradiation. We resolved the atomic arrangement to be a close-packed stacking structure taking planar shapes of equilateral triangles, isosceles trapezoids or parallelograms with internal angles of $60^{\circ}$ or $120^{\circ}$. The change in morphology was realized by local atomic shuffling, which suggests that the solid-solid transformation hypothesis is correct. We also note that this technique can be applied to investigations of other clusters or even single atoms or molecules.

\section{Methods}

Sample preparation. Single-layer graphene was grown on a $25 \mu \mathrm{m}$-thick copper foil (Alfa Aesar, item No. 13382) in a flow-type low-pressure reactor. The recipe was adapted from ${ }^{37}$. The substrate was heated to $1035^{\circ} \mathrm{C}$ under a pressure of $640 \mathrm{mTorr}$ with a mixed gas flow of $\mathrm{CH}_{4} / \mathrm{H}_{2}$. The graphene transferring technique was adapted from $^{38}$. Monolayer or bilayer graphene was obtained by transferring to the TEM grid once or twice. More details about the characterization and the recipe can be found in the supporting materials of Reference 18 . The sample was heated to $400^{\circ} \mathrm{C}$ under vacuum for the purpose of cleaning before deposition of the Fe clusters. The deposition was realized in a pulsed laser deposition (PLD) chamber with a background pressure of 100 mTorr. The graphene transferred to the TEM grid was placed in the upright position to the target a distance of $100 \mathrm{~mm}$. A pulsed highpower laser ( $400 \mathrm{~mJ} /$ pulse and $40 \mathrm{~ns} /$ pulse) was used to eject particles from the target surface, which were then deposited on the free-standing graphene.

TEM imaging and simulations. TEM imaging was carried out using an aberrationcorrected and monochromated FEI Titan 80-300 microscope with a typical electron beam current density of about $7 \times 10^{6} \mathrm{e} \cdot \mathrm{s}^{-1} \cdot \mathrm{nm}^{-2}\left(\sim 100 \mathrm{~A} / \mathrm{cm}^{2}\right)$. The third-order spherical aberration was carefully tuned to about $1 \mu \mathrm{m}$. The microscope was operated at $60 \mathrm{kV}$ to minimize the knock-on damage to the graphene. Since the point resolution and the information limit were more sensitive to the chromatic aberration at lower operation voltages, the gun monochromator has been excited to 1.8 to reduce the electron energy spread to $<0.2 \mathrm{eV}$. Images were recorded on a charge coupled device (CCD) camera $(2 \mathrm{k} \times 2 \mathrm{k}$, Gatan UltraScanTM 1000) with a binning two mode. The read-out time was $0.5 \mathrm{~s}$. The HRTEM images were taken with an exposure time of $2 \mathrm{~s}$ and a spatial sampling of $0.20 \AA$ / pixel. HRTEM image simulations were performed using the commercial software MacTempas. The input microscope parameters were: an acceleration voltage of $60 \mathrm{kV}$, a spherical aberration of $1 \mu \mathrm{m}$, a chromatic aberration of $1.5 \mathrm{~mm}$, a focal spread of $2.5 \mathrm{~nm}$ and a convergence angle of $0.1 \mathrm{mrad}$.

1. Baletto, F. \& Ferrando, R. Structural properties of nanoclusters: Energetic, thermodynamic, and kinetic effects. Reviews of Modern Physics 77, 371-423 (2005).

2. Yacaman, M. J., Ascencio, J. A., Liu, H. B. \& Gardea-Torresdey, J. Structure shape and stability of nanometric sized particles. Journal of Vacuum Science \& Technology B: Microelectronics and Nanometer Structures 19, 1091-1103 (2001).

3. Smith, D. J., Petfordlong, A. K., Wallenberg, L. R. \& Bovin, J. O. Dynamic AtomicLevel Rearrangements in Small Gold Particles. Science 233, 872-875 (1986).

4. Hansen, P. L. et al. Atom-Resolved Imaging of Dynamic Shape Changes in Supported Copper Nanocrystals. Science 295, 2053-2055 (2002).

5. Marks, L. D. Experimental studies of small particle structures. Reports on Progress in Physics 57, 603 (1994).

6. Barnard, A. S. Modelling of nanoparticles: approaches to morphology and evolution. Reports on Progress in Physics 73, 086502 (2010).

7. Vollath, D. \& Fischer, F. D. Fluctuations, bistability and hysteresis connected to phase transformations of nanoparticles. Progress in Materials Science 56, 1030-1076 (2011)

8. Koga, K., Ikeshoji, T. \& Sugawara, K.-i. Size- and Temperature-Dependent Structural Transitions in Gold Nanoparticles. Physical Review Letters 92, 115507 (2004).
9. Iijima, S. \& Ichihashi, T. Structural instability of ultrafine particles of metals. Physical Review Letters 56, 616-619 (1986).

10. Ben-David, T. et al. Correlated Orientations in Nanocrystal Fluctuations. Physical Review Letters 78, 2585-2587 (1997).

11. Ajayan, P. M. \& Marks, L. D. Experimental evidence for quasimelting in small particles. Physical Review Letters 63, 279-282 (1989).

12. Li, Z. Y. et al. Three-dimensional atomic-scale structure of size-selected gold nanoclusters. Nature 451, 46-48 (2008).

13. van Huis, M. A. et al. Atomic Imaging of Phase Transitions and Morphology Transformations in Nanocrystals. Advanced Materials 21, 4992-4995 (2009).

14. Lee, S., Fan, C., Wu, T. \& Anderson, S. L. CO Oxidation on Aun/TiO2 Catalysts Produced by Size-Selected Cluster Deposition. J. Am. Chem. Soc. 126, 5682-5683 (2004).

15. Qiao, B. et al. Single-atom catalysis of CO oxidation using Pt1/FeOx. Nat. Chem. 3, 634-641 (2011)

16. Meyer, J. C., Girit, C. O., Crommie, M. F. \& Zettl, A. Imaging and dynamics of light atoms and molecules on graphene. Nature 454, 319-322 (2008).

17. Wang, H. et al. Interaction between single gold atom and the graphene edge: A study via aberration-corrected transmission electron microscopy. Nanoscale 4, 2920-2925 (2012).

18. Wang, H. et al. Doping Monolayer Graphene with Single Atom Substitutions. Nano Lett. 12, 141-144 (2011).

19. Wang, H. et al. Dynamics of Metal Clusters on Graphene Edge. In submission (2012).

20. Besley, N. A., Johnston, R. L., Stace, A. J. \& Uppenbrink, J. Theoretical study of the structures and stabilities of iron clusters. Journal of Molecular Structure: THEOCHEM 341, 75-90 (1995).

21. Schebarchov, D. \& Hendy, S. C. Transition from Icosahedral to Decahedral Structure in a Coexisting Solid-Liquid Nickel Cluster. Physical Review Letters $\mathbf{9 5}$, 116101 (2005).

22. Lin, M. E., Ramachandra, A., Andres, R. P. \& Reifenberger, R. Electronic states and structural stability of supported Au clusters. Zeitschrift für Physik D Atoms, Molecules and Clusters 26, 59-63 (1993).

23. Khandelwal, G. S. \& Merzbacher, E. Displacement Cross Sections for Fast Electrons Incident on Gold. Physical Review 130, 1822-1825 (1963).

24. Williams, D. \& Carter, B. Transmission Electron Microscopy: A Textbook for Materials Science. (2004).

25. Zhu, W., Wang, H. \& Yang, W. Evolution of graphene nanoribbons under lowvoltage electron irradiation. Nanoscale 4, 4555-4561 (2012).

26. Girit, Ç. Ö. et al. Graphene at the Edge: Stability and Dynamics. Science 323, 1705-1708 (2009).

27. Schaäffel, F. et al. Atomic Resolution Imaging of the Edges of Catalytically Etched Suspended Few-Layer Graphene. ACS Nano 5, 1975-1983 (2011).

28. Datta, S. S., Strachan, D. R., Khamis, S. M. \& Johnson, A. T. C. Crystallographic Etching of Few-Layer Graphene. Nano Letters 8, 1912-1915 (2008).

29. Booth, T. J. et al. Discrete Dynamics of Nanoparticle Channelling in Suspended Graphene. Nano Letters 11, 2689-2692 (2011).

30. Ramasse, Q. M. et al. Direct Experimental Evidence of Metal-Mediated Etching of Suspended Graphene. ACS Nano 6, 4063-4071 (2012).

31. Zan, R., Bangert, U., Ramasse, Q. \& Novoselov, K. S. Interaction of Metals with Suspended Graphene Observed by Transmission Electron Microscopy. The Journal of Physical Chemistry Letters 3, 953-958 (2012).

32. Boukhvalov, D. W. \& Katsnelson, M. I. Destruction of graphene by metal adatoms. Applied Physics Letters 95, 023109 (2009).

33. Ferrando, R., Rossi, G., Nita, F., Barcaro, G. \& Fortunelli, A. Interface-Stabilized Phases of Metal-on-Oxide Nanodots. ACS Nano 2, 1849-1856 (2008).

34. Tian, W. et al. Hexagonal close-packed Ni nanostructures grown on the (001) surface of MgO. Applied Physics Letters 86, 131915-131913 (2005).

35. Barcaro, G., Fortunelli, A., Rossi, G., Nita, F. \& Ferrando, R. Epitaxy, Truncations, and Overhangs in Palladium Nanoclusters Adsorbed on $\mathrm{MgO}(001)$. Physical Review Letters 98, 156101 (2007).

36. Piotrowski, M. J., Piquini, P. \& Da Silva, J. L. F. Density functional theory investigation of 3d, 4d, and 5d 13-atom metal clusters. Physical Review B 81, 155446 (2010).

37. Li, X. S. et al. Large-Area Synthesis of High-Quality and Uniform Graphene Films on Copper Foils. Science 324, 1312-1314 (2009).

38. Regan, W. et al. A direct transfer of layer-area graphene. Appl. Phys. Lett. 96, 113102-113103 (2010)

\section{Acknowledgments}

HT Wang and W Yang are grateful for financial support from the National Science Foundation of China (Grant No. 10832009; Grant No. 11090333) and the Science Foundation of Chinese University (Grant No. 2011QNA4038).

\section{Author contributions}

XX Zhang coordinated the project. XX Zhang and HT Wang conceived and designed the experiment. YB Yao performed PLD and sputtering experiment. QX Wang performed TEM together with K Li. HT Wang and W Yang grew graphene and prepared TEM samples. HT 
Wang, XX Zhang, U Schwingenschlögl, Y Chen and W Yang wrote and revised the manuscript. All authors discussed about the results and commented on the manuscript.

\section{Additional information}

Supplementary information accompanies this paper at http://www.nature.com/ scientificreports
Competing financial interests: The authors declare no competing financial interests. License: This work is licensed under a Creative Commons

Attribution-NonCommercial-NoDerivs 3.0 Unported License. To view a copy of this license, visit http://creativecommons.org/licenses/by-nc-nd/3.0/

How to cite this article: Wang, H.T. et al. Unraveling the Atomic Structure of Ultrafine Iron Clusters. Sci. Rep. 2, 995; DOI:10.1038/srep00995 (2012). 